\title{
A U87-EGFRvIII cell-specific aptamer mediates small interfering RNA delivery
}

\author{
XINGMEI ZHANG ${ }^{1}$, HUIYU LIANG ${ }^{1}$, YAN TAN $^{1}$, XIDONG WU $^{1}$, SHUJI LI $^{1}$ and YUSHENG SHI ${ }^{2}$ \\ ${ }^{1}$ Department of Neurobiology, School of Basic Medical Sciences; ${ }^{2}$ Department of Radiation Oncology, \\ Nanfang Hospital, Southern Medical University, Guangzhou, Guangdong 510515, P.R. China
}

Received March 10, 2014; Accepted April 24, 2014

DOI: $10.3892 /$ br.2014.276

\begin{abstract}
U87-EGFRvIII is a U87 glioma cell line that overexpresses epidermal growth factor receptor variant III (EGFRvIII). In the present study, we investigated whether a DNA aptamer selected against U87-EGFRvIII using cell-based systematic evolution of ligands by exponential enrichment (cell-SELEX) could deliver c-Met small interfering RNA (siRNA) into U87-EGFRvIII cells and silence the targeted gene expression. The selected biotinylated aptamer (BA) was coupled to biotinylated c-Met siRNA by streptavidin to deliver siRNA into U87-EGFRvIII cells. c-Met siRNA, transfected with lipofectamine 2000, served as a positive control, while control siRNA, transferred with BA, served as a negative control. Western blotting was performed to detect changes in the c-Met protein expression, and MTT and Annexin V-fluorescein isothiocyanate/propidium iodine assays were used to determine changes in the proliferation and apoptosis of U87-EGFRvIII cells, respectively. Similar to the liposome-mediated group, U87-EGFRvIII cells that were transfected with BA-c-Met siRNA experienced a significant decrease in the c-Met protein expression $(\mathrm{P}<0.05)$. There were also significant increases in the apoptotic rate $(\mathrm{P}<0.05)$ and inhibition rate of cell growth $(\mathrm{P}<0.01)$ compared with the negative control group, indicating that BA could deliver c-Met siRNA into U87-EGFRvIII and result in target gene silencing. In conclusion, the results demonstrated that this DNA aptamer, obtained through cell-SELEX, can be used as an efficient and targeted carrier for siRNA delivery, providing a novel approach and strategy for the targeted combination therapy of glioblastoma.
\end{abstract}

\footnotetext{
Correspondence to: Dr Yusheng Shi, Department of Radiation Oncology, Nanfang Hospital, Southern Medical University, 1838 Guangzhou Avenue North, Baiyun, Guangzhou, Guangdong 510515, P.R. China

E-mail: zxmray@hotmail.com
}

Key words: aptamer, siRNA delivery, c-Met, EGFRvIII, glioblastoma

\section{Introduction}

Small interfering RNAs (siRNAs) are intermediate products in RNA interference (RNAi) pathways that can cause target gene silencing via RNAi pathways (1). Traditional siRNA delivery vehicles fail to address the instability and poor bioavailability of siRNAs and are associated with low delivery efficiency, poor uptake by target cells and immunotoxicity for protein carriers. Effective siRNA delivery to target systems without causing damage to normal cells or tissue remains the biggest challenge for in vivo studies (2). Aptamers are oligonucleotides (single-stranded DNA or RNA) that bind to target molecules with a high affinity and good specificity and can be obtained using the systematic evolution of ligands by exponential enrichment (SELEX) system (3). This technique adopts random oligonucleotide libraries of $10^{13}-10^{15}$ molecules and exponentially enriches oligonucleotides that bind to the target molecule specifically via polymerase chain reaction amplification. This technique, when used to select aptamers by targeting living cells, is known as cell-SELEX (4), which offers more advantages over protein-based SELEX. Cell-SELEX obviates the requirement of protein purification and immobilization, allows target molecules to maintain their natural spatial structure and keeps protein glycosylation sites on the cell surface in a natural state. Aptamers obtained in this way can bind target molecules in their natural state and can be directly used for cell identification and detection.

Glioblastoma multiforme (GBM) is the most common and aggressive human malignant brain tumor, accounting for $50 \%$ of solid brain tumors. A common feature of patients with GBM is the presence of an increased expression and mutation of human epidermal growth factor receptor (EGFR), with the most common mutant being EGFR variant III (EGFRvIII). EGFRvIII is produced from the deletion of 801 base pairs in exons 2-7 of the extracellular domain of EGFR and is closely associated with the progression of GBM and its resistance to radiotherapy and chemotherapy. Varying degrees of a high EGFRvIII expression are found in other malignancies, including non-small cell lung cancer and colorectal cancer. However, EGFRvIII is not expressed in normal tissue (5). Therefore, EGFRvIII can be harnessed as a specific target for tumor therapy. c-Met is encoded by proto-oncogene c-Met and is a tyrosine kinase receptor of hepatocyte growth factor (HGF). In GBM patients, overexpression of c-Met often leads 
to poor prognosis, and the expression of c-Met is significantly higher in recurrent GBM compared with primary GBM (6).

Thus far, aptamers that have been designed for siRNA delivery are mainly aimed at prostate cancer (7-9), AIDS (10-12) and breast cancer (13). We have previously studied the selection of high-affinity and high-specificity aptamers targeting a U87MG glioma cell line with EGFRvIII overexpression (U87-EGFRvIII). One of these aptamers, designated as 32, was internalized and located in the nucleus of U87-EGFRvIII cells (14). In the present study, aptamer 32 was biotinylated and coupled to biotin-labeled c-Met siRNA via streptavidin, and this delivered c-Met siRNA into U87-EGFRvIII cells in a targeted manner. Subsequently, significant changes in the c-Met protein expression, apoptosis and proliferation of U87-EGFRvIII cells were observed.

\section{Materials and methods}

Aptamer and siRNA. The 5'-biotin-labeled aptamer 32, termed BA, was synthesized by Invitrogen Life Technologies (Carlsbad, CA, USA). The sequence was as follows: biotin-5'-GCAATG GTACGGTACTTCCTGAATGTTGTTTTTTCTCTTT TCTCTATAGTACAAAAGTGCACGCTACTTTGCTAA-3'. The c-Met siRNA sequence (synthesized by Ribo Biotech Co. Ltd., Guangzhou, Guangdong, China) was 5'-AGC CAAUUUAUCAGGAGGUTT-3' (sense); and 5'-ACCUCC UGAUAAAUUGGCUTT-3' (antisense). The negative control siRNA was Cy3-labeled siR-Ribo ${ }^{\mathrm{TM}}$ Transfection Control (siN05815122144).

Cell culture. U87MG cells were cultured in Dulbecco's modified Eagle's medium (DMEM) containing 10\% fetal bovine serum (FBS), $100 \mathrm{U} / \mathrm{ml}$ penicillin and $100 \mu \mathrm{g} / \mathrm{ml}$ streptomycin (P/S). The U87-EGFRvIII cell line (15) (obtained from Dr Webster Cavenee, Ludwig Cancer Institute, San Diego, CA, USA) was grown in DMEM supplemented with $10 \%$ FBS, $1 \%$ $\mathrm{P} / \mathrm{S}$ and $100 \mathrm{mg} / \mathrm{ml}$ geneticin. The cells were cultured at $37^{\circ} \mathrm{C}$ in a $5 \% \mathrm{CO}_{2} / 95 \%$ air environment.

Lipofectamine 2000 transfection and BA-mediated delivery. The U87-EGFRvIII cells were seeded in 12-well plates at a density of $1 \times 10^{5}$ cells/well. The following day, the cells were harvested for transfection at 30-50\% confluence. The media were switched to $1 \mathrm{ml}$ of serum-free, antibiotic-free media prior to transfection. A total of $5 \mu \mathrm{l} /$ well of $20 \mu \mathrm{M}$ BA and $5 \mu \mathrm{l} /$ well of $20 \mu \mathrm{M}$ biotin-labeled c-Met siRNA were added into $100 \mu \mathrm{l}$ of Opti-MEM (Gibco-BRL, Carlsbad, CA, USA) respectively, gently pipetted 3-5 times and allowed to stand for $5 \mathrm{~min}$. Subsequently, the two solutions were mixed with $2.5 \mu \mathrm{l} /$ well of $20 \mu \mathrm{M}$ streptavidin (Sigma-Aldrich, St. Louis, MO, USA) and left to stand for $20 \mathrm{~min}$ prior to being added to the culture media. The transfection system was $200 \mu \mathrm{l}$, and the final concentration of BA and siRNA was $100 \mathrm{nM}$. A total of $5 \mu \mathrm{l} /$ well of liposome (Invitrogen Life Technologies) and $5 \mu \mathrm{l} /$ well of c-Met siRNA plus $2.5 \mu \mathrm{l} /$ well of streptavidin served as a positive control and underwent the same aforementioned procedures. BA + control siRNA + streptavidin and liposome + control siRNA + streptavidin were used as negative controls for BA and liposome, respectively. Separate groups exposed to BA or streptavidin alone were provided to exclude the interference from BA or streptavidin, respectively. Following transfection, the cells were cultured in an incubator $\left(37^{\circ} \mathrm{C}, 5 \% \mathrm{CO}_{2}\right)$ for $6 \mathrm{~h}$, following which the cells were cultured for another $72 \mathrm{~h}$ in DMEM containing $10 \% \mathrm{FBS}$.

Western blotting. Cells were washed with phosphate-buffered saline (PBS) $72 \mathrm{~h}$ after transfection and lysed in cell lysis buffer containing $1 \%$ protease inhibitor. The protein content was measured using the bicinchoninic acid method. Following denaturation, the samples $(10 \mu \mathrm{g} /$ well $)$ were subjected to $8 \%$ SDS-PAGE, transferred to a polyvinylidene fluoride membrane, and incubated with rabbit anti-human c-Met (Santa Cruz Biotechnology, Inc., Santa Cruz, CA, USA) and rabbit anti-human GAPDH antibodies (Santa Cruz Biotechnology, Inc.) at $4^{\circ} \mathrm{C}$ overnight prior to exposure to horseradish peroxidase-labeled secondary antibody at room temperature for $1 \mathrm{~h}$. Target proteins were visualized using enhanced chemiluminescence detection and analyzed with Fluorchem SP imaging system (Alpha Innotech Corporation, San Leandro, CA, USA).

Annexin V-fluorescein isothiocyanate/propidium iodine (PI) assay. The cells were washed with PBS and digested with $0.25 \%$ EDTA-free trypsin $72 \mathrm{~h}$ after transfection. Following termination of the digestion, the cell suspension was transferred to a $1.5 \mathrm{ml}$ Eppendorf tube and centrifuged at $100 \mathrm{x} \mathrm{g}$ for $5 \mathrm{~min}$. The supernatant was discarded, while the cell pellets were resuspended in PBS (500 $\mu \mathrm{l} /$ tube) and centrifuged as aforementioned. Following the addition of $200 \mu$ l detection buffer to each tube, $2 \mu \mathrm{l}$ of Annexin V and $2 \mu \mathrm{l}$ of PI (Nanjing KeyGen Biotech Co., Ltd., Nanjing, Jiangsu, China) were added in sequence. The resultant mixture was incubated on ice in the dark for $10 \mathrm{~min}$ before performing flow cytometry to detect the percentage of apoptotic cells. To prove the targeting specificity of BA for U87-EGFRvIII cells, U87MG cells were transfected as control cells in this experiment and for the MTT assays.

MTT colorimetric assay. The cells were seeded in 24-well plates at a density of $5 \times 10^{4}$ cells/well, with each well containing $500 \mu \mathrm{l}$ of cell suspension. The cells were switched to serum-free medium, $72 \mathrm{~h}$ after transfection. Subsequently, $50 \mu \mathrm{l}$ of MTT $(5 \mathrm{mg} / \mathrm{ml})$ was added to each well and was incubated at $37^{\circ} \mathrm{C}$ for $4 \mathrm{~h}$, the supernatant was discarded, while the cell pellets were exposed to $500 \mu \mathrm{l}$ of dimethyl sulfoxide and incubated at $37^{\circ} \mathrm{C}$ for $15 \mathrm{~min}$. Optical absorbance (OD) at $570 \mathrm{~nm}$ was measured on a microplate reader.

Statistical analysis. Data were presented as the means \pm SD. One-way analysis of variance was performed using SPSS 13.0 statistical software (SPSS, Inc., Chicago, IL, USA). P $<0.05$ was considered to indicate a statistically significant difference.

\section{Results}

BA-mediated delivery of c-Met siRNA into U87-EGFRvIII cells downregulates $c$-Met protein expression. An agarose gel electrophoresis analysis was performed to identify the amount of BA, c-Met siRNA and streptavidin used for conjugation. When the final ratio for BA, streptavidin and siRNA was 2:1:2, a single and distinct band was obversed (Fig. 1A), suggesting 
A

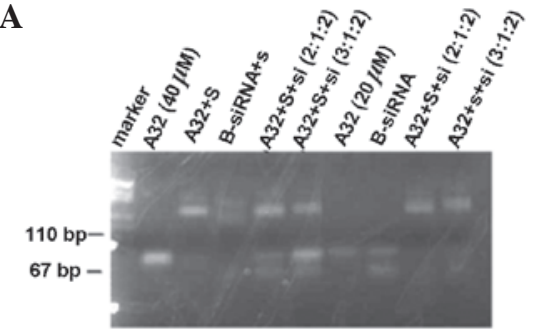

B

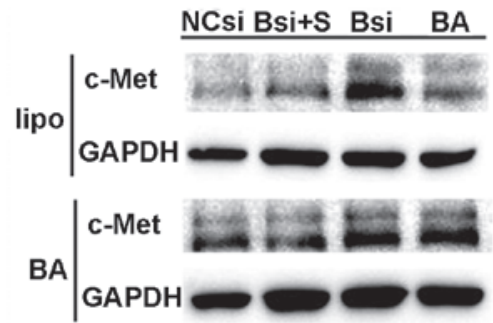

C

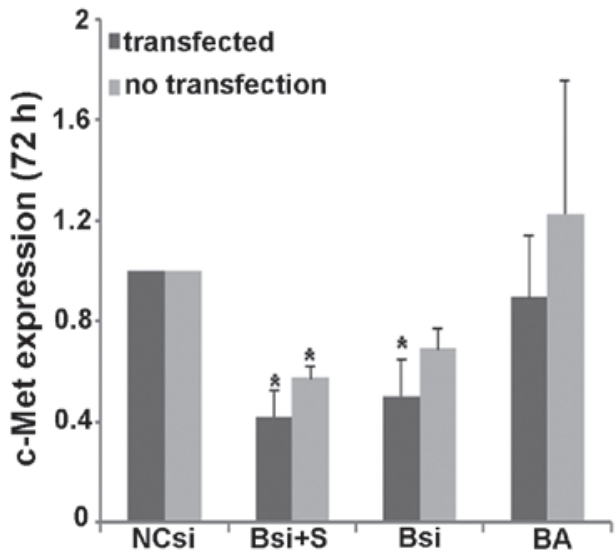

Figure 1. BA-mediated delivery of c-Met siRNA into U87-EGFRvIII cells downregulated c-Met protein expression. (A) Determination of the amount of BA, c-Met siRNA (small interfering RNA) and streptavidin used for conjugation was performed by agarose gel electrophoresis. A32, biotin-aptamer 32 (BA); B-siRNA, biotin-c-Met siRNA; B-siRNA + s = biotin-c-Met siRNA + streptavidin. A32 + S + si = biotin-A32 + streptavidin + biotin-c-Met siRNA. (B) Western blotting and (C) quantitative analysis of the changes in c-Met protein expression following BA-delivered c-Met siRNA into U87-EGFRvIII cells. Transfected and lipo, liposome-mediated group; no transfection and BA group, BA-mediated group; NCsi = liposome or BA + control siRNA + streptavidin; $\mathrm{Bsi}+\mathrm{S}=$ liposome or BA + c-Met siRNA + streptavidin; Bsi = liposome or BA + c-Met siRNA; BA = BA alone. Error bars show the means \pm standard deviation $(n=3) .{ }^{*} \mathrm{P}<0.05$.

that it was the optimal ratio for BA, streptavidin and siRNA conjugation. Western blotting results showed that $72 \mathrm{~h}$ after transfection, with a c-Met siRNA final concentration of $100 \mathrm{nM}$, the c-Met protein expression in the liposome- and BA-mediated groups was significantly reduced compared with cells transfected with control siRNA $(0.418 \pm 0.18, \mathrm{P}=0.026$; $0.57 \pm 0.09, \mathrm{P}=0.039$ ) (Fig. 1B-C). The presence of streptavidin or BA alone had no effect on the protein levels of c-Met. The liposome-mediated siRNA delivery downregulated the target gene expression by $>50 \%$. Similarly, BA-mediated siRNA delivery reduced the target gene expression by $\sim 50 \%$. The results showed that BA mediated the delivery of siRNA into U87-EGFRvIII cells, leading to target gene silencing.

c-Met gene silencing as a result of BA-mediated $c$-Met siRNA delivery increases apoptosis of glioma cells. The percentage of apoptotic glioma cells was determined using flow cytometry. The results showed that in the U87-EGFRvIII cells, the liposome- and BA-mediated groups had an apoptotic rate of $26.47 \pm 7.49$ and $23.23 \pm 10.14 \%$, respectively, revealing a significant increase over their respective negative control group $(\mathrm{P}=0.011$ and $\mathrm{P}=0.025)$. There was no difference in the apoptotic rate between the liposome- and BA-mediated groups $(\mathrm{P}=0.573)$. However, in the U87MG cells, the apoptotic rate of the liposome-mediated group $(19.1 \pm 5.15 \%)$ increased significantly compared with that of its control group $(\mathrm{P}=0.004)$ and differed significantly from the BA-mediated group $(8.33 \pm 3.3 \%)(\mathrm{P}=0.006)$. The apoptotic rate of the BA-mediated group exhibited no difference from its control group ( $\mathrm{P}=0.991)$ (Fig. 2). These results indicated that BA specifically mediated the delivery of c-Met siRNA into U87-EGFRvIII cells but not U87MG cells, and therefore, reduced c-Met gene expression and induced U87-EGFRvIII apoptosis.
c-Met gene silencing as a result of BA-mediated c-Met siRNA delivery decreases the proliferation of glioma cells. The OD values of each group were determined with the MTT assays. Similar to the flow cytometry results, in the U87-EGFRvIII cells the inhibition rate of the liposome- and BA-mediated groups was $61.77 \pm 6.73$ and $68.43 \pm 5.87 \%$, respectively, showing a significant increase compared with that of the respective control groups $(\mathrm{P}<0.01$ and $\mathrm{P}<0.01)$. The inhibition rate did not differ between the two groups $(\mathrm{P}=0.762)$. In the U87MG cells, the inhibition rate of the liposome-mediated group $(45.78 \pm 10.53 \%)$ increased significantly compared with its control group and the BA-mediated group $(6.46 \pm 1.78 \%)$ $(\mathrm{P}<0.01$ and $\mathrm{P}<0.01$, respectively). The inhibition rate of the BA-mediated group showed no difference from the control group ( $\mathrm{P}=0.971)$ (Fig. 3). These findings demonstrated that the BA-mediated c-Met siRNA delivery into U87-EGFRvIII cells suppressed U87-EGFRvIII cell proliferation while producing no impact on that of U87MG cells.

\section{Discussion}

GBM patients have extremely poor prognosis and short median survival rates (16). EGFR-targeting protein tyrosine kinase inhibitors are highly immunogenic and are associated with various degrees of resistance, leading to the failure of cancer chemotherapy. The type and level of biomarkers varies in different types of tumors with different degrees of differentiation and location. Aptamers selected against living cells by cell-SELEX can recognize specific cell surface receptors and can therefore be used to identify tumor cell surface biomarkers $(4,17)$. Following binding to surface proteins on target cells, certain aptamers are internalized within cells (18-20). The advantages of aptamers over conventional antibodies include low molecular weight, rapid synthesis 
A

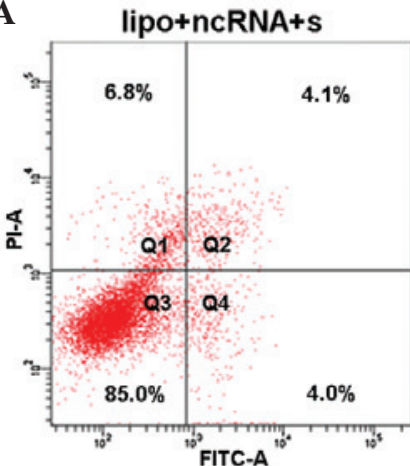

C

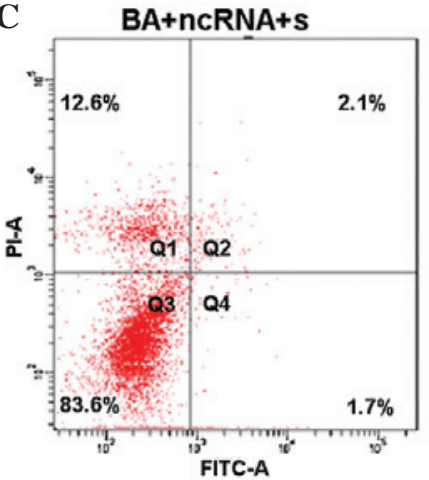

B

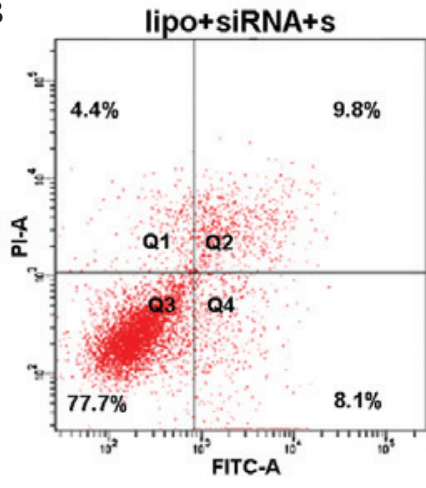

D

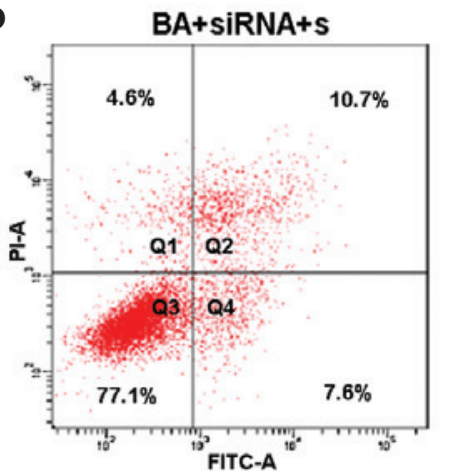

$\mathbf{E}$

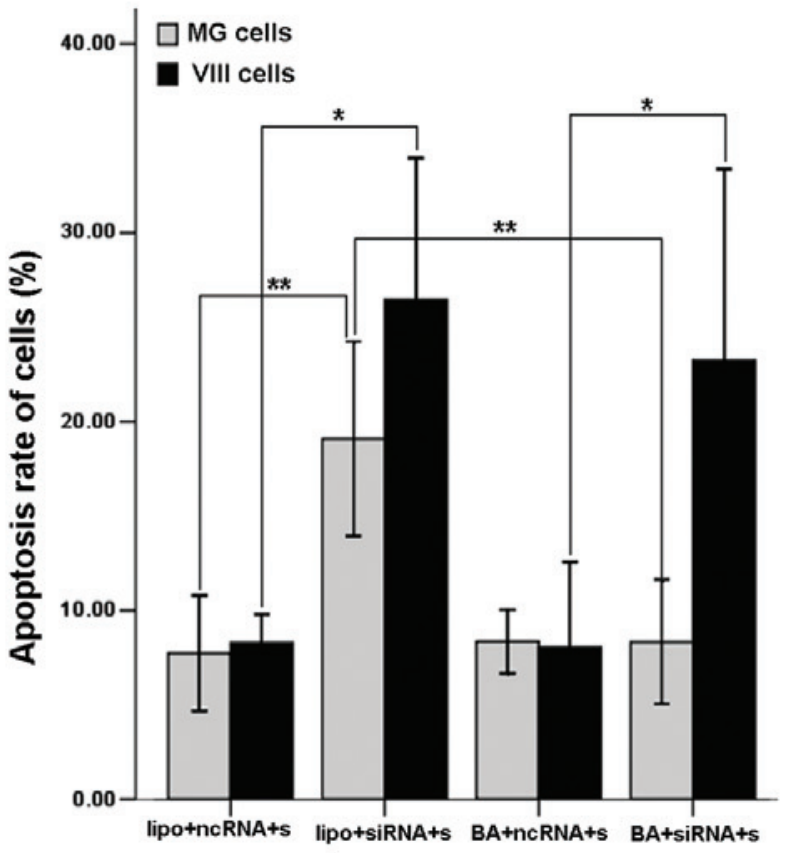

Figure 2. Flow cytometry detection of apoptotic changes of U87-EGFRvIII cells following c-Met gene silencing. (A-D) Flow cytometry results of U87-EGFRvIII apoptosis in each group. Q2 and Q4 show late and early apoptotic rates, respectively. (E) Comparison of apoptotic rate of U87MG and U87-EGFRvIII cells in each group. BA, 5'-biotin-labeled aptamer 32; FITC, fluorescein isothiocyanate; siRNA, small interfering RNA; lipo + ncRNA + s = liposome + control siRNA + streptavidin. lipo + siRNA + s = liposome + c-Met siRNA + streptavidin; BA + ncRNA + s = BA + control siRNA + streptavidin; $\mathrm{BA}+\operatorname{siRNA}+\mathrm{s}=\mathrm{BA}+\mathrm{c}-$ Met siRNA + streptavidin. Error bars show the means \pm standard deviation $(\mathrm{n}=3) .{ }^{*} \mathrm{P}<0.05,{ }^{* *} \mathrm{P}<0.01$.

in vitro, low toxicity, low immunogenicity, good tissue permeability and ease of chemical modification, which minimizes degradation and enhances pharmacokinetic parameters in vivo. There are two key issues in the application of RNAi in the clinical trials of human diseases. The first issue is an effective means for siRNA delivery, and the second issue is the specificity of siRNA-targeted delivery and the safety of the delivery vehicle in humans. The aforementioned characteristics of aptamers make them promising siRNA delivery vehicles (21). An aptamer for human epidermal growth factor receptor 2 (HER2) can carry siRNA into HER2-expressing breast cancer cells to mediate target gene silencing (13). Aptamers targeting human immunodeficiency virus (HIV) glycoprotein 120 and cluster of differentiation 4 can also mediate the delivery of their corresponding siRNAs into target cells in the form of aptamer-siRNA chimeras, thereby inhibiting HIV-1 viral replication $(10,11)$. In the present study, an aptamer obtained through a cell-SELEX procedure mediated the delivery of siRNA into glioma cells with EGFRvIII overexpression, specifically silenced target genes and modulated the apoptosis and proliferation of U87-EGFRvIII cells. This aptamer did not affect the survival rate of U87MG cells as it did not bind and enter the U87MG cells. In a previous report, we proved that EGFRvIII is the target receptor of BA (14), and we therefore hypothesized that receptor-mediated endocytosis is a possible mechanism for siRNA delivery. The present study provides evidence for the feasibility of aptamers as siRNA delivery vehicles.

Abnormal HGF/c-Met signals can lead to the stimulation of tumor cell functions, including cell proliferation, survival, cell

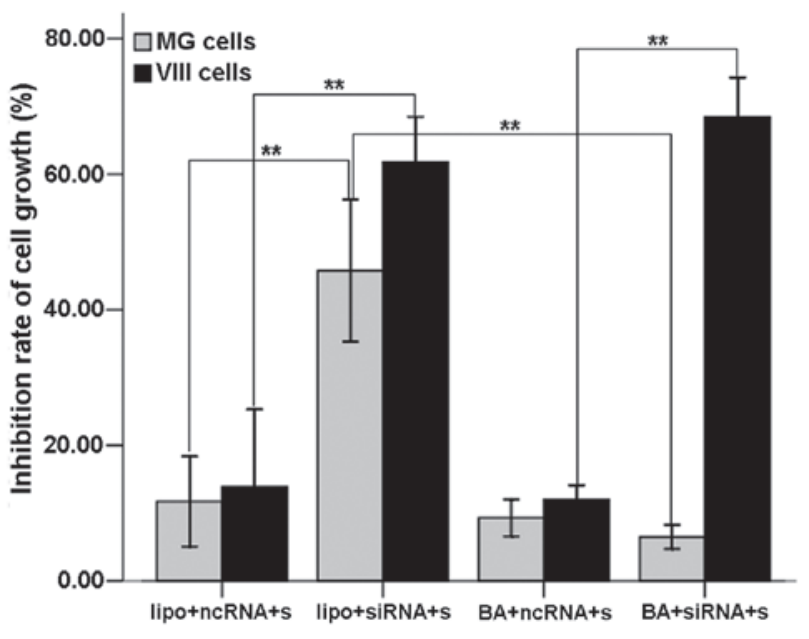

Figure 3. Comparison of the inhibitory rate on the proliferation of U87MG and U87-EGFRvIII cells following c-Met gene silencing. The inhibitory rate was calculated according to the OD values of the control and experimental groups obtained from the MTT assay. OD, optical density; BA, 5'-biotin-labeled aptamer 32; siRNA, small interfering RNA; lipo + ncRNA + s = liposome + control siRNA + streptavidin; lipo + siRNA + s = liposome + c-Met siRNA + streptavidin; BA + ncRNA + s = BA + control siRNA + streptavidin; $\mathrm{BA}+$ siRNA + s = BA + c-Met siRNA + streptavidin. Data show the means \pm standard deviation of three independent experiments. ${ }^{* *} \mathrm{P}<0.01$.

dispersion and movement, epithelial to mesenchymal transition, angiogenesis, invasion and metastasis $(22,23)$. HGF/c-Met overexpression and EGFRvIII high expression in gliomas are closely associated with the development and progression of 
gliomas, complicating the diagnosis and treatment of gliomas. The synergy among these molecules attenuates the response of gliomas to any single chemotherapeutic agent and tends to induce drug resistance as a result. It has been reported that the overexpression of c-Met can activate HER3 and cause resistance to the chemotherapeutic agent, fibrate gemfibrozil, which is an EGFR inhibitor used in the treatment of patients with lung cancer (24). The combination of c-Met and EGFR kinase inhibitors can enhance the therapeutic effect on EGFRvIII-positive GBM and overcome its chemoresistance (25). In glioma cells overexpressing EGFRvIII, c-Met activation is not dependent on its ligand HGF, but is dependent on the activation of the EGFRvIII-mediated tyrosine kinase pathway. This indicates that the c-Met receptor may be another significant target for the treatment of EGFRvIII-positive GBM. In the case of a high EGFRvIII expression, c-Met is also highly activated in tumors and is detrimental to chemotherapy in cancer patients (26). In the present study, c-Met gene silencing inhibited EGFRvIII overexpressed glioma cell proliferation and induced apoptosis, indicating that the co-suppression of HGF/c-Met and EGFRvIII signaling pathways can be more effective in glioma treatment. Notably, aptamer-mediated c-Met siRNA delivery into U87-EGFRvIII cells offers a combined treatment regimen for patients with a high expression of c-Met and EGFRvIII.

Chu et al (7) created a biotin-labeled anti-prostate-specific membrane antigen (PSMA) aptamer and a biotin-labeled siRNA, which were incubated with streptavidin containing four binding sites. The siRNA, streptavidin and aptamer formed stable conjugates via the association between streptavidin and biotin, resulting in target gene silencing in PSMA-positive cells. Biotinylated aptamers link to biotinylated siRNA via a streptavidin bridge, offering a simple yet effective approach for siRNA delivery. In the present study, c-Met expression did not differ in the presence or absence of streptavidin during liposomal transfection of c-Met siRNA, and the addition of streptavidin alone had no effect on the expression of c-Met, indicating that streptavidin has no cytotoxicity. Future studies should examine the role of aptamer-mediated c-Met siRNA delivery in tumor suppression in an animal model of glioma.

\section{Acknowledgements}

The present study was supported by grants from the National Science Foundation of China (grant nos. 30973481 and 81272509) and the Guangdong Natural Science Foundation (grant nos. 9151051501000053 and grant no. 07005145).

\section{References}

1. Elbashir SM, Harborth J, Lendeckel W, Yalcin A, Weber K and Tuschl T: Duplexes of 21-nucleotide RNAs mediate RNA interference in cultured mammalian cells. Nature 411: 494-498, 2001.

2. Burnett JC and Rossi JJ: RNA-based therapeutics: current progress and future prospects. Chem Biol 19: 60-71, 2012.

3. Tuerk $\mathrm{C}$ and Gold L: Systematic evolution of ligands by exponential enrichment: RNA ligands to bacteriophage T4 DNA polymerase. Science 249: 505-510, 1990.
4. Sefah K, Shangguan D, Xiong X, O'Donoghue MB and Tan W: Development of DNA aptamers using Cell-SELEX. Nature Protoc 5: 1169-1185, 2010.

5. Gan HK, Kaye AH and Luwor RB: The EGFRvIII variant in glioblastoma multiforme. J Clin Neurosci 16: 748-754, 2009.

6. Liu W, Fu Y, Xu S, et al: c-Met expression is associated with time to recurrence in patients with glioblastoma multiforme. J Clin Neurosci 18: 119-121, 2011.

7. Chu TC, Twu KY, Ellington AD and Levy M: Aptamer mediated siRNA delivery. Nucleic Acids Res 34: e73, 2006.

8. McNamara JO II, Andrechek ER, Wang Y, et al: Cell type-specific delivery of siRNAs with aptamer-siRNA chimeras. Nat Biotechnol 24: 1005-1015, 2006.

9. Dassie JP, Liu XY, Thomas GS, et al: Systemic administration of optimized aptamer-siRNA chimeras promotes regression of PSMA-expressing tumors. Nat Biotechnol 27: 839-849, 2009.

10. Zhou J, Li H, Zhang J, Piotr S and Rossi J: Development of cell-type specific anti-HIV gp120 aptamers for siRNA delivery. J Vis Exp 52: 2954, 2011.

11. Wheeler LA, Trifonova R, Vrbanac V, et al: Inhibition of HIV transmission in human cervicovaginal explants and humanized mice using CD4 aptamer-siRNA chimeras. J Clin Invest 121: 2401-2412, 2011

12. Zhou J, Swiderski P, Li H, et al: Selection, characterization and application of new RNA HIV gp 120 aptamers for facile delivery of Dicer substrate siRNAs into HIV infected cells. Nucleic Acids Res 37: 3094-3109, 2009.

13. Thiel KW, Hernandez LI, Dassie JP, et al: Delivery of chemo-sensitizing siRNAs to HER2+-breast cancer cells using RNA aptamers. Nucleic Acids Res 40: 6319-6337, 2012.

14. Tan Y, Shi YS, Wu XD, et al: DNA aptamers that target human glioblastoma multiforme cells overexpressing epidermal growth factor receptor variant III in vitro. Acta Pharmacol Sin 34: 1491-1498, 2013.

15. Zhan Y and O'Rourke DM: SHP-2-dependent mitogen-activated protein kinase activation regulates EGFRvIII but not wild-type epidermal growth factor receptor phosphorylation and glioblastoma cell survival. Cancer Res 64: 8292-8298, 2004

16. Nicholas MK, Lukas RV, Chmura S, Yamini B, Lesniak M and Pytel P: Molecular heterogeneity in glioblastoma: therapeutic opportunities and challenges. Semin Oncol 38: 243-253, 2011.

17. Shangguan D, Cao ZC, Li Y and Tan W: Aptamers evolved from cultured cancer cells reveal molecular differences of cancer cells in patient samples. Clin Chem 53: 1153-1155, 2007.

18. Huang YF, Shangguan D, Liu H, et al: Molecular assembly of an aptamer-drug conjugate for targeted drug delivery to tumor cells. Chembiochem 10: 862-868, 2009.

19. Wu Y, Sefah K, Liu H, Wang R and Tan W: DNA aptamer-micelle as an efficient detection/delivery vehicle toward cancer cells. Proc Natl Acad Sci USA 107: 5-10, 2010.

20. Zhang K, Sefah K, Tang L, et al: A novel aptamer developed for breast cancer cell internalization. ChemMedChem 7: 79-84, 2012.

21. Thiel KW and Giangrande PH: Intracellular delivery of RNA-based therapeutics using aptamers. Ther Deliv 1: 849-861, 2010.

22. McCarty JH: Glioblastoma resistance to anti-VEGF therapy: has the challenge been MET? Clin Cancer Res 19: 1631-1633, 2013.

23. Peruzzi B and Bottaro DP: Targeting the c-Met signaling pathway in cancer. Clin Cancer Res 12: 3657-3660, 2006.

24. Engelman JA, Zejnullahu K, Mitsudomi T, et al: MET amplification leads to gefitinib resistance in lung cancer by activating ERBB3 signaling. Science 316: 1039-1043, 2007.

25. Pillay V, Allaf L, Wilding AL, et al: The plasticity of oncogene addiction: implications for targeted therapies directed to receptor tyrosine kinases. Neoplasia 11: 448-458, 2 p following 458, 2009.

26. Huang PH, Mukasa A, Bonavia R, et al: Quantitative analysis of EGFRvIII cellular signaling networks reveals a combinatorial therapeutic strategy for glioblastoma. Proc Natl Acad Sci USA 104: 12867-12872, 2007. 